\title{
Post-functionalization of Ether-linked Polymer via the Application of Ullmann-coupling Reaction: Synthesis, Characterization and Thermal Degradation Kinetics
}

\author{
Suha S. Al-Tarawneh ${ }^{* a}$ and Taher S. Ababneh ${ }^{\text {b }}$ \\ ${ }^{a}$ Department of Chemistry and Chemical Technology, Tafila Technical University, Tafila \\ 66110, Jordan. \\ ${ }^{b}$ Chemistry Department, Yarmouk University, Irbid 21163, Jordan.
}

Received: $10^{\text {th }}$ Feb. 2021; $\quad$ Accepted: $15^{\text {th }}$ April 2021

\begin{abstract}
A new ether-linked polymer (PE-A) was synthesized via the polycondensation of 1,4-dibromo-2,5-difluorobenzene with biphenol $(\mathrm{A})$. The new polymer has shown a good solubility in non-polar solvents, as well as moderate thermal stability (up to $300{ }^{\circ} \mathrm{C}$ ). The parent PE-A was subjected to post-functionalization modification applying the Ullmann-coupling reaction on the $\mathrm{C}$-Br bonds of $\mathrm{PE}-\mathrm{A}$, where the bromo-sites have been replaced by aniline-linked moieties. The resulting polymer (PE-Ani-A) shows slight solubility in several solvents. Its thermal stability was enhanced by $36 \%$ when compared with PE-A. To ensure the significant impact of the inclusion of the aniline group on thermal stability, a thermal degradation kinetics study was performed and the CoatsRedfren and Broido theoretical models were applied to explore the degradation process. The calculated activation energy for PE-Ani-A degradation was thereby found to be higher than that of PE-A, which indicates the higher thermal stability of PE-Ani-A. For further insights into the thermal stability of the polymers, the limited oxygen index (LOI), which represents the flame-retardant property of the polymers, was calculated. The results indicate that PE-A is a promising candidate as a flame-retardant polymer ( $\mathrm{LOI}=46.7$ ), since it has a high bromo-content. On the other hand, PE-Ani-A has a lower LOI although it has a higher thermal stability.
\end{abstract}

Keywords: Ether-linked polymers, Ullmann-coupling reaction, Thermal degradation kinetics.

\section{Introduction}

Aromatic polyethers or ether-based polymers are high-performance polymers that gained an appreciated interest in the material science field due to their excellent thermal stability, good electrical properties and solvent and oxidative resistance ${ }^{[1-2]}$. However, they are known for lack of solubility, which contributes to poor processability and casting as films ${ }^{[2]}$. From the aforementioned properties, the thermal stability is an essential requirement for most recent applications at elevated conditions. Ether-linked polymers are known for their outstanding thermal stability, as the polymer structure combines high contents of halogens, aromatic and heterocyclic linkers ${ }^{[3-5]}$. The presence of these linkers enhances rigidity, cross-linking and crystallinity, which have pronounced influence on the thermal stability of polymers. On the other hand, the inclusion of halogen substituents, such as $-\mathrm{Cl}$ and $-\mathrm{Br}$ groups, plays a role toward designing polymers with good flame-retardant properties $^{[0-7]}$. The capability of the polymers to be flame-retardant can be determined from the thermal gravimetric analysis measurements (TGA) and the value of the limited oxygen index $(\mathrm{LOI})^{[8-9]}$ which is defined as the minimum amount of oxygen (\%) that will sustain the degradation process. When the LOI value 
exceeds 28 , then the polymer is considered as a good flame-retardant candidate. Examples on these polymers include poly(brominated) polymers, poly(ether-amine), poly(aminopyridines), poly(ether-sulfone), poly(ether-imide), poly(imnies) with LOI values of $49,33,32,35,46$ and 32 , respectively ${ }^{[10-13]}$. The high LOI values support the fact that the incorporation of halogens or heterocycles within an ether-linked chain improves the thermal stability and the flame-retardancy of polymers. This variation is related to the changes in the polymer structure and the molecular architecture of the chains ${ }^{[14]}$. With this respect, more attention is paid to the determination of the activation energy $\left(E_{a}\right)$ of the polymer degradation process which can be extracted from the thermal degradation kinetics monitored by gravimetric analysis measurements (TGA) by applying relevant mathematical models ${ }^{[15-16]}$.

The theoretical background of the thermal degradation study is depicted in literature by applying different mathematical approximations to Equation 1.1. All reported calculations are differentiated according to different sets of parameters. For example, some methods are model-free, iso-conversional methods performed at different heating rates and constant conversion, while they do not deal with the reaction mechanism. These methods include, but are not limited to, Freidman, Flynn-Wall-Ozawa, Chang, Kissinger and others ${ }^{[15,17,20]}$. In contrast, other methods can explore the mechanism of the degradation upon applying several fitting calculations of the function $\mathrm{g}(\alpha)$, where the activation energy is determined from the best linear correlation coefficient. Examples on these methods are the Coats-Redfern and the Broido methods ${ }^{[14]}$.

$$
\frac{d \alpha}{d t}=k(T) g(\alpha)
$$

$\mathrm{T}$ : absolute temperature in $\mathrm{K}, \alpha$ : conversion, $t$ : time, $k$ : reaction rate constant, $g(\alpha)$ : the conversion function or reaction model.

\section{Coats-Redfern Method (CR-Method)}

The CR-method is a non-isothermal integral method that assumes that the activation energy does not depend on the degree of conversion. It is represented by the linear function of time at a constant heating rate $(b)$ as depicted by equation 1.2, which is derived from the asymptotic approximation and reviewed by Johnson and Gallagher $^{[21]}$.

$$
\frac{\ln g(\alpha)}{T^{2}}=\frac{\ln (A R)}{\beta E}-\frac{E_{a}}{R T}
$$

$A$ is thereby the pre-exponential Arrhenius factor and $R$ is the general gas constant. The activation energy can be calculated from the linear slope of plotting $\ln g(\alpha) / T^{2}$ vs $1 / T$. By assuming various reaction orders $(\mathrm{n}=0,1 / 2,1 / 3$, $2 / 3, \ldots$ ) fitted on different kinetic models $g(\alpha)$, the CR-method can be represented by equations 1.3 and 1.4:

$$
\ln \left[\frac{1-(1-\alpha)^{1-n}}{T^{2}(1-n)}\right]=\ln \left[\left(\frac{A R}{\beta E_{a}}\right)\left(1-\frac{2 R T}{E_{a}}\right)\right]-\frac{E_{a}}{R T} \quad \text { for } n \neq 1
$$

For the first order reactions, the above equation modifies to eq. 1.4:

$$
\ln \left[\frac{-\ln (1-\alpha)}{T^{2}}\right]=\ln \left[\left(\frac{A R}{\beta E_{a}}\right)\left(1-\frac{2 R T}{E_{a}}\right)\right]-\frac{E_{a}}{R T} \quad \text { for } n=1
$$

The bracket $\left(1-2 R T / E_{a}\right)$ is assumed to be approximately equal to 1 . The fraction of the decomposed sample $(\alpha)$ is represented by equation 1.5:

$$
\alpha=\frac{w_{0}-w_{t}}{w_{0}-w_{f}}
$$

$w_{0}$ is thereby the initial weight of the sample, $w_{f}$ is the final weight and $w_{t}$ is the weight at a given time of the experiment.

\section{The Broido Method}

This method has been developed by Broido to evaluate the activation energy associated with each stage of decomposition according to equation 1.6. A plot of $(\ln (-\ln (1-\alpha))$ vs. $1 / T$ gives an excellent approximation to a straight line, where the slope is related to the activation energy $^{[21]}$.

$$
\ln (-\ln (1-\alpha))=-\frac{E_{a}}{R T}+K
$$


In this work, we report the synthesis of a new poly(brominated-ether)-linked polymer and its post-functionalization by aniline through the catalytic Ullmann-coupling reaction ${ }^{[17]}$. The thermal stability, LOI and activation energy of the original as well as the post-functionalized polymers will be determined and compared applying the above discussed CR and Broido methods.

\section{Materials and Methods}

All chemicals were purchased from SigmaAldrich and used without further purification unless otherwise noted. All synthesis work and post-functionalization steps were performed under a nitrogen atmosphere using the Schlenk line technique. Carbon, hydrogen, oxygen and nitrogen analysis was performed using a Vario EL elemental analyzer. Thermogravimetric analysis (TGA) was carried out using a TA Instruments Q-5000IR series thermal gravimetric analyzer with samples held in $50 \mu \mathrm{L}$ platinum pans under an atmosphere of air (heating rate 10 $\left.{ }^{\circ} \mathrm{C} / \mathrm{min}\right)$. Scanning electron microscopy imaging (SEM) was taken on a Hitachi SU-70 scanning electron microscope. IR spectra were recorded on a Shimadzu FT-IR-8300 in transmission mode in form of $\mathrm{KBr}$ pellets in the wavenumber range $4000-400 \mathrm{~cm}^{-1}$.

\section{Synthesis of Polymers}

\section{Bromo-substituted Ether-based Polymer (PE-A)}

The polycondensation of 1,4-dibromo-2,5difluorobenzene (F-Ar-F) with biphenol was carried out in a one-pot procedure. To a solution of F-Ar-F (1.6 mmol) and biphenol derivative (1.6 mmol) in $15 \mathrm{~mL}$ dimethylacetamide (DMAc), potassium carbonate $(4 \mathrm{mmol})$ was added and the solution was allowed to warm up to $130^{\circ} \mathrm{C}$ for 20 hours under nitrogen flow. After polymerization, the product was poured into $100 \mathrm{~mL}$ of $3 \%$ hydrochloric acid and the polymer precipitated as a white solid with $77 \%$ yield and dried under vacuum at room temperature for 24 hours. The prepared polymer was confirmed by the elemental analysis of $\mathrm{C}, \mathrm{H}$ and $\mathrm{O}$. Calculated for $\mathrm{C}_{18} \mathrm{H}_{10} \mathrm{Br}_{2} \mathrm{O}: \mathrm{C}, 53,77 \%$; H, 2.51\%; Br, 39.74\%; O, $3.98 \%$. Found: C, $51.22 \%, \mathrm{H}, 2.77 \%$; $\mathrm{O}, 3.82 \%$; $\mathrm{Br} \%$ was not measured.
Bromo-substituted Ether-based Polymer (PEAni-A)

The post-functionalization of PE-A was performed by applying the Ullmann-coupling reaction between the bromide substituent of PEA $(5 \mathrm{mmol})$ and a mixture of aniline $(7.5 \mathrm{mmol})$, potassium carbonate $(10 \mathrm{mmol})$, copper iodide $(0.5 \mathrm{mmol})$ and L-proline $(1 \mathrm{mmol})$ in $10 \mathrm{~mL}$ of dimethyl sulfoxide (DMSO). The mixture was heated up to $90{ }^{\circ} \mathrm{C}$ for 24 hours under nitrogen flow. The cooled mixture was partitioned between water and ethyl acetate. A pale-brown solid was filtered, washed with $0.1 \mathrm{M} \mathrm{NaOH}, 0.1$ $\mathrm{M} \mathrm{HCl}$, water and acetone. The final product with $82 \%$ yield was dried under vacuum at room temperature for 24 hours. The prepared polymer was confirmed by elemental analysis. Calculated for $\mathrm{C}_{24} \mathrm{H}_{16} \mathrm{NO}: \mathrm{C}, 86.20 \%$; $\mathrm{H}, 4.82 \%$; $\mathrm{N}, 4.19 \%$; $\mathrm{O}, 4.78 \%$. Found: $\mathrm{C}, 85.11 \%$; $\mathrm{H}, 4.55 \%$; $\mathrm{O}$, $3.67 \%$.

\section{Results and Discussion}

The ether-based polymer poly (dibromobenzene-biphenoxy $)_{n}$ (PE-A) has been prepared via a one-step polycondensation reaction between 1,4-dibromo-2,5-difluorobenzene and biphenol in the presence of potassium carbonate as a base (Scheme 1). After the formation of the bis-phenoxide basic sites, a nucleophilic substitution reaction on the $\mathrm{C}-\mathrm{F}$ bond takes place and yields the extended-ether bonds ${ }^{[2]}$. The polymer was washed several times with water to dissolve any residual potassium fluoride salt. The final product is a white solid isolated in a good yield (77\%) and is soluble in common nonpolar solvents, such as diethyl ether and THF. Upon dissolving, clear, colorless and flexible films were formed when cast from the solution. The formation of the polymer was confirmed by the elemental analysis of carbon, hydrogen and oxygen, while no data was collected for bromine and fluorine. The characteristic ether-link bond formation $(\mathrm{C}-\mathrm{O}-\mathrm{C})$ was confirmed by infrared spectroscopy as evident from the absorption band at $1355 \mathrm{~cm}^{-1}$ corresponding to the C-O-C bond (Figure 1). Moreover, the condensation is confirmed by the absence of the $\mathrm{OH}$-stretching characteristic bands above $3000 \mathrm{~cm}^{-1}$ (Figure 1).

Post-functionalization of PE-A has been achieved applying the Ullmann-coupling reaction between the aryl-halide part of the polymer and aromatic primary amine ${ }^{[11,18]}$. 


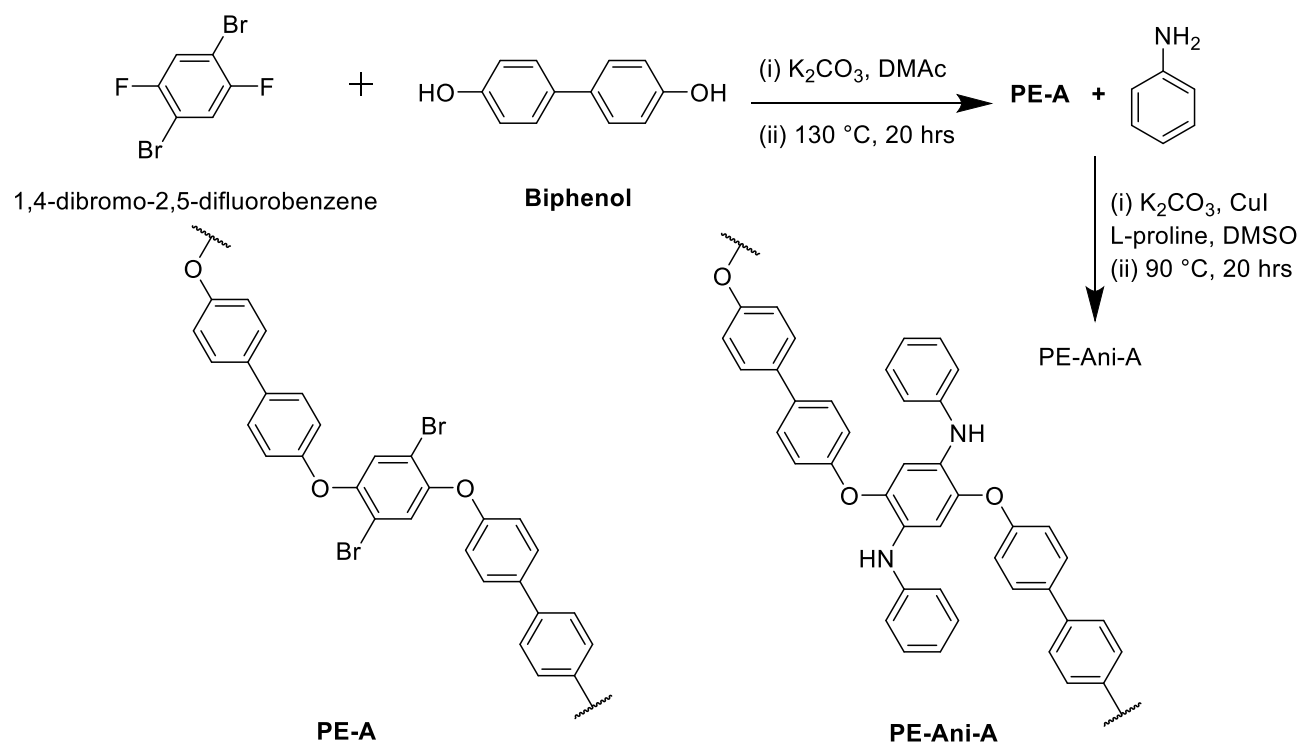

Scheme 1. Molecular structures of the monomers and the polymers PE-A and PE-Ani-A.

Herein, the bromo-sites of the PE-A chain react with aniline in presence of copper iodide as a catalyst and L-proline (amino acid) as a ligand (Scheme 1). The synthesized functionalized polymer was washed several times with water, acid, base and acetone to give poly(dibromobenzene-biphenoxy) $\mathrm{n}_{\mathrm{n}}$ as a stable brown solid (PE-Ani-A). PE-Ani-A is soluble in non-polar organic solvents and slightly soluble in methanol. This enhanced solubility is

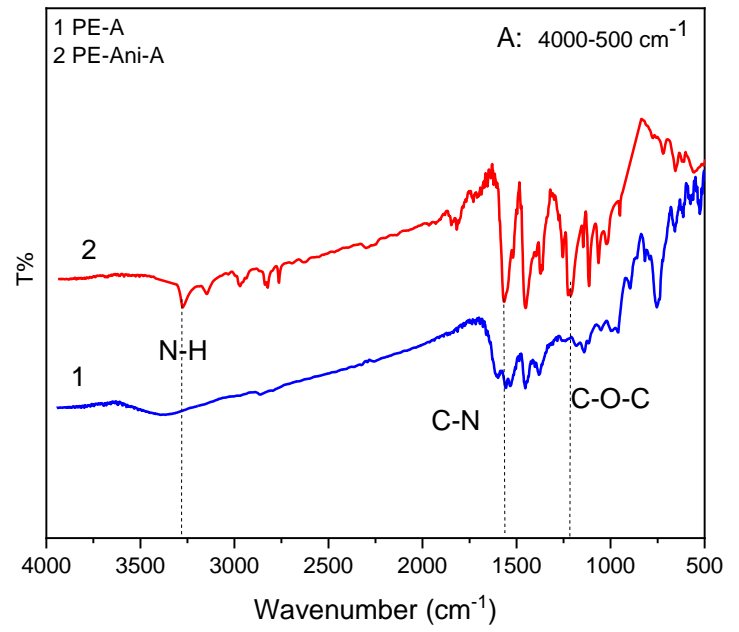

attributed to the presence of the free N-H bond of linked aniline. The successful formation of the post-functionalized polymer (PE-Ani-A) was confirmed by $\mathrm{CHO}$ elemental analysis. In addition to the bands of the parent polymer PEA, the IR spectrum of PE-Ani-A shows a new absorption band around $3300 \mathrm{~cm}^{-1}$ corresponding to the stretch vibration of the N-H bond (Figure 1).

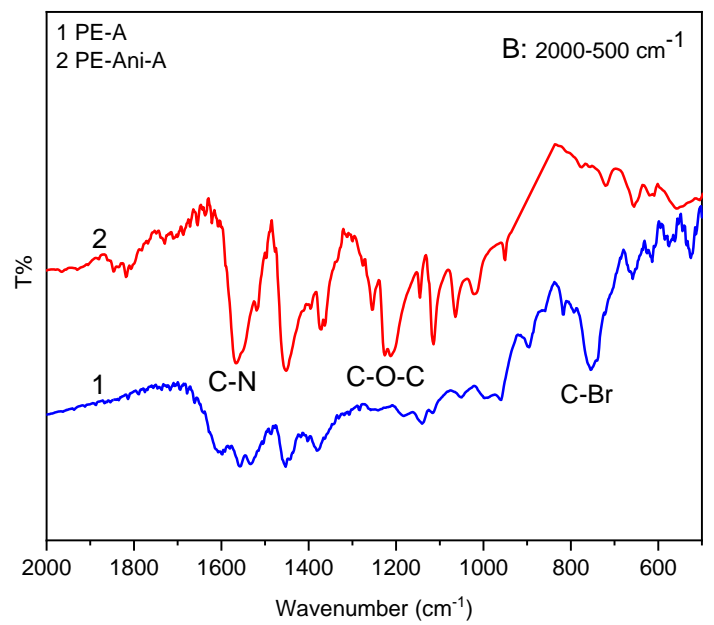

Figure 1. IR spectra of PE-A and PE-Ani-A in the range A) $4000-500 \mathrm{~cm}^{-1}$ and B) $2000-500 \mathrm{~cm}^{-1}$.

SEM measurements were used to establish the surface morphology of prepared polymers (Figure 2). PE-A shows thereby a random aggregated morphology. However, there is a notable well-ordered behavior of the surface upon post-functionalization, represented by the formation of random aggregated irregular spherical particles $0.1-0.3 \mu \mathrm{m}$ in size (Figure 2). This change in the surface morphology also indicates the rapid and random impact of the polymerization and the crosslinking across the polymer framework ${ }^{[19]}$. Furthermore, the more defined surface might be attributed to the 

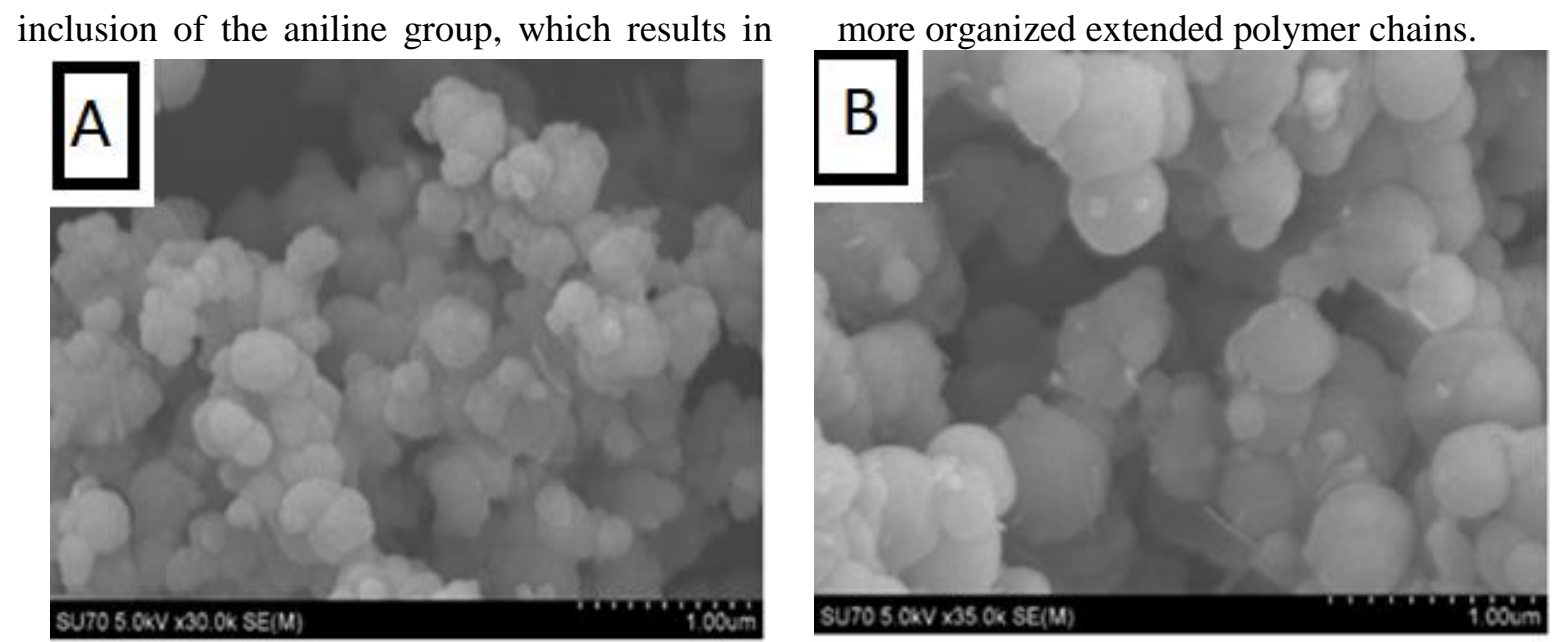

Figure 2. Scanning Electron micrographs of A) PE-A and B) PE-Ani-A.

The thermal degradation behavior of the solid-state polymers was evaluated by interpreting the TGA curves ${ }^{[20]}$. In this report, the thermal properties of the synthesized polymers; PE-A and the post-functionalized polymer PE-Ani-A were evaluated by TGA over the temperature range $30-600{ }^{\circ} \mathrm{C}$ at a heating rate of $10 \mathrm{~K} / \mathrm{min}$ and under nitrogen atmosphere (Figure 3). The TGA profile of PE-A shows that it is thermally stable up to $300{ }^{\circ} \mathrm{C}$. However, there is a pronounced enhancement of the thermal stability of PE-Ani-A (up to $400{ }^{\circ} \mathrm{C}$ ) over PE-A. This difference is attributed to changes in the polymer structure. In general, PE-A reveals aromatic moieties functionalized with bromo-sites and

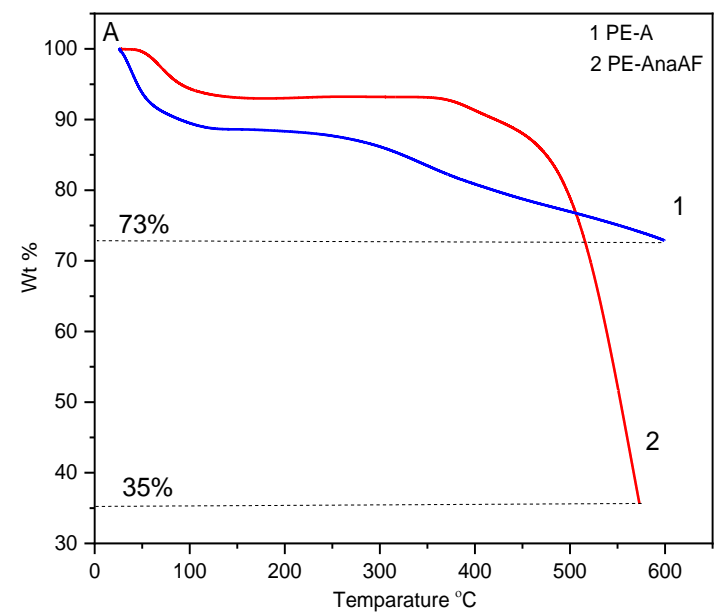

connected via ether linkages. On the other hand, PE-Ani-A has a similar structural content of PEA with the bromo-sites being replaced by aromatic secondary amine groups (aniline groups). Thus, the incorporation of aniline groups in PE-Ani-A enriched the polymer structure with higher aromatic and nitrogen contents compared to PE-A. These play a vital role in increasing the rigidity of the polymer and hence its thermal stability ${ }^{[16]}$. In addition, it is well-known that a higher percent composition of hetero-atoms (nitrogen in this case) plays a significant role in increasing the thermal stability and lowering the rate of the thermal degradation process.

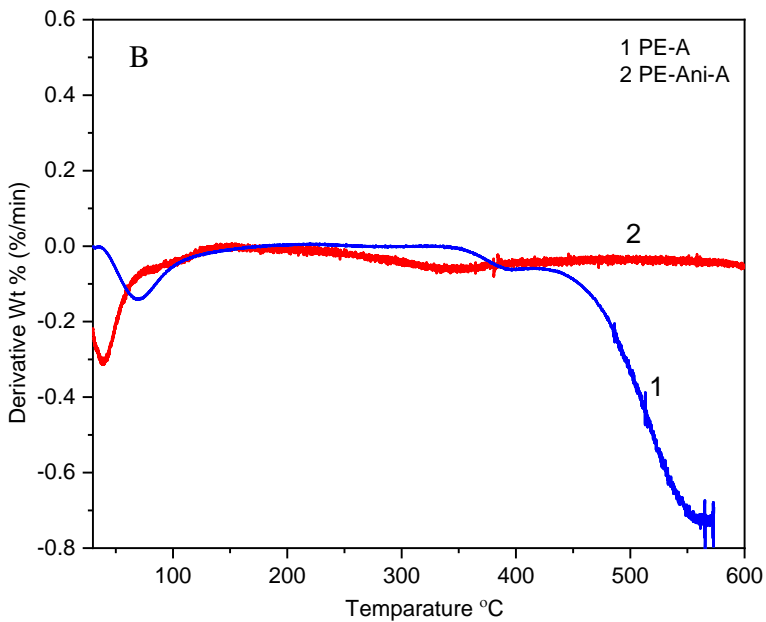

Figure 3. A) TGA curves of PE-A and PE-Ani-A and B) the corresponding DTG curves; (heating rate $10 \mathrm{~K} / \mathrm{min})$.

From the TGA curves, the residual weight (so-called char residue) can also be determined. The char residue represents the percent of polymer weight remaining at the end of the process. PE-A has shown a significant amount of char residue (73\%), which can be attributed to the presence of bromo-sites. On the other hand, the char residue of PE-Ani-A (35\%) is half the value of PE-A. It is well-known that halogen atoms resist the oxidation or degradation of 
polymers at elevated temperatures, which enhances their applicability as flame-retardant materials. The flame-retardant property can be estimated from the LOI value which can be calculated from the char residue using the Van Krevelen's correlation ${ }^{[9]}$.

$$
L O I=17.5+0.4 \times(\text { Char residue }) \quad \text { Eq. } 17
$$

If the calculated value of LOI is higher than 28 , the polymer can be cons idered as a flameretardant material, while below this value, the polymers are considered to be flammable. The estimated LOI values for PE-A and PE-Ani-A are 31.5 and 46.7, respectively. Both polymers are obviously good candidates as flame-retardant polymers, which can be attributed to the role of $\mathrm{Br}$ in PE-A and the presence of nitrogen in PEAni-A. The higher LOI for PE-A is due to the role of halogens in this regard.
For further investigations, the stages of decomposition of the polymers were determined from their thermogravimetric derivative (DTG) curves (Figure 3B). The DTG curves of both polymers show one common degradation stage around $100{ }^{\circ} \mathrm{C}$, which represents the weight loss related to the evaporation of water due to moisture. The other stages are related to the degradation of the contents of polymers. These stages appear in the ranges of $250-400{ }^{\circ} \mathrm{C}$ and 340-450 ${ }^{\circ} \mathrm{C}$ for PE-A and PE-Ani-A, respectively. The higher degradation stage of PE-Ani$\mathrm{A}$ is a further supportive indication of its notable higher thermal stability. Table 1 shows summarized data that represents the weight loss of the polymers, char residue, the temperatures at $50 \%$ and $100 \%$ degradation (T50, T100) and the peak temperature (Ts), which represents the midtemperature of each degradation stage.

Table 1. Interpretation data of TGA and DTG curves.

\begin{tabular}{cccccc}
\hline Polymer & Stage $\left({ }^{\circ} \mathbf{C}\right)$ & $\mathbf{T}_{\mathbf{s}}\left({ }^{\circ} \mathbf{C}\right)$ & $\mathbf{W t} \%$ & $\mathbf{C R} \%$ & LOI \\
PE-A & S: $250-400$ & 350 & 27 & 73 & 46.7 \\
PE-Ani-A & S: $350-450$ & 400 & 65 & 35 & 31.5 \\
\hline
\end{tabular}

$\mathrm{S}$ : Degradation stage, $\mathrm{T}_{\mathrm{s}}$ : The peak temperature derived from the DTG curve, Wt\%: Weight loss, CR: Char residue, LOI: Limited oxygen index.

\section{Kinetic Parameters}

TGA is a well-known useful technique to extrapolate the kinetic parameters from the thermal degradation process. The kinetic parameters involve the activation energy $\left(E_{a}\right)$, reaction order (n) and pre-exponential factor (A). Their values depend on the rate and nature of the flowing atmosphere during the measurement, the heating rate and the sample mass.

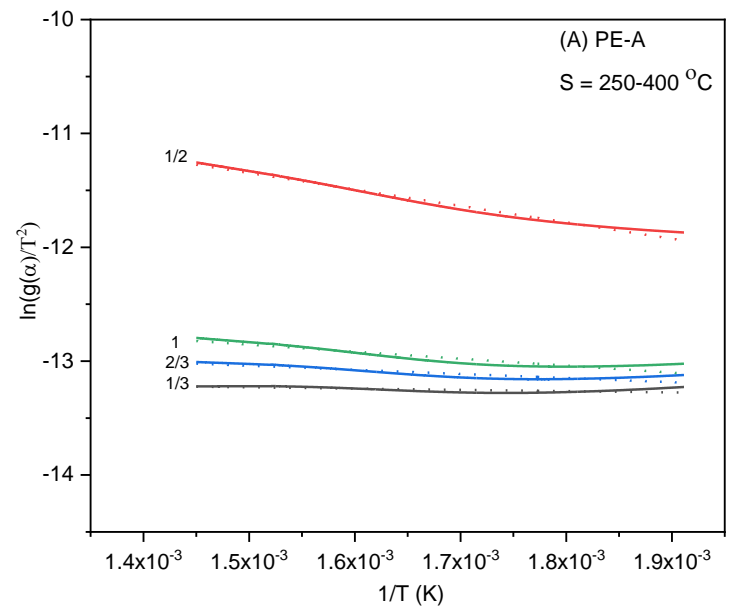

The activation energy $\left(E_{a}\right)$ was estimated for the two polymers using the CR-method, adopting the reaction-order models which have been discussed in Eqs. 1.3 and 1.4. Different reaction orders were assumed $(n=1,1 / 2,1 / 3,2 / 3)$ with a constant heating rate $\left(10{ }^{\circ} \mathrm{C} / \mathrm{min}\right)$. The plots of $\left[\ln g(\alpha) / T^{2}\right]$ vs. (1/T) for each polymer at different $n$ values and at the stage of degradation (S) are shown in Figure 4.

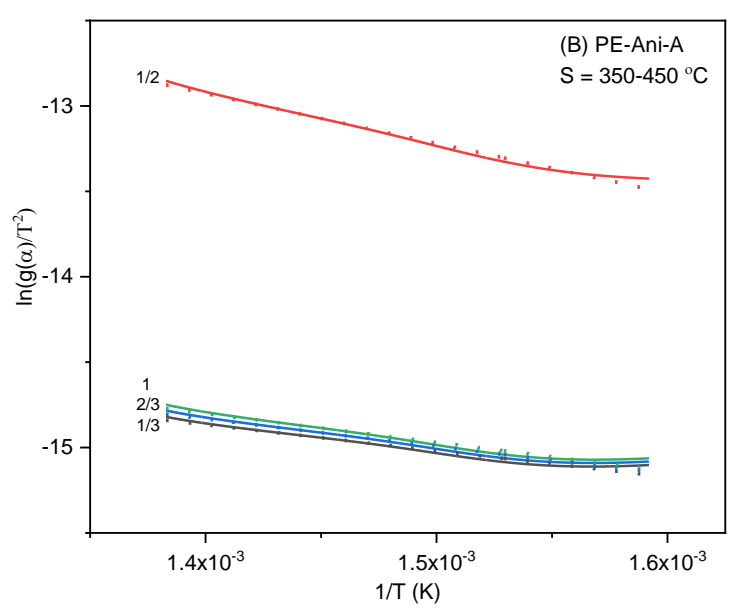

Figure 4. CR-method model and the fitting parameters of the polymers at different reaction orders and at the degradation stage (S): A) PE-A and B) PE-Ani-A. 
The values of $E_{a}$ and $R^{2}$ obtained for the different reaction orders are summarized in Table 2. Best fitting and maximum $\mathrm{R}^{2}$ values were obtained for both polymers for the reaction order $n=1 / 2$. At $n=1 / 2$, the calculated activation energies of the polymers PE-A and PE-Ani$\mathrm{A}$ are 12.0 and $24.3 \mathrm{~kJ} / \mathrm{mol}$, respectively. The higher value for $E_{a}$ in the case of PE-Ani-A is a further indication of its high thermal stability, as more energy is required to activate the degradation. The low to moderate activation energy values for these polymers might be attributed to both the variation in the aromatic moieties and the presence of ether linker. Thus, the proposed degradation mechanism might reveal the degradation to start at the ether bond point in PE-A, resulting in a lower $E_{a}$ than for PE-Ani-A where degradation occurs at the aniline-linkage.

Table 2. Activation energy by the CR-method corresponding to selected reaction order (n) and degradation stage.

\begin{tabular}{|c|c|c|c|c|c|}
\hline \multirow[t]{2}{*}{ Polymer } & \multirow{2}{*}{$\begin{array}{c}\text { Degradation } \\
\text { Stage } \\
\left({ }^{\circ} \mathrm{C}\right)\end{array}$} & \multicolumn{4}{|c|}{$\begin{array}{c}\mathbf{E}_{\mathbf{a}}(\mathbf{k J} / \mathbf{m o l}) \\
\quad\left(\mathbf{R}^{2}\right)\end{array}$} \\
\hline & & $n=1 / 3$ & $n=1 / 2$ & $n=2 / 3$ & $\mathrm{n}=\mathbf{1}$ \\
\hline PE-A & S: $250-400$ & $\begin{array}{c}0.93 \\
(0.9590)\end{array}$ & $\begin{array}{c}12.0 \\
(0.9842)\end{array}$ & $\begin{array}{c}2.99 \\
(0.8984)\end{array}$ & $\begin{array}{c}5.11 \\
(0.9827)\end{array}$ \\
\hline PE-Ani-A & S: $350-450$ & $\begin{array}{c}12.7 \\
0.9728 \\
\end{array}$ & $\begin{array}{c}24.3 \\
0.9908 \\
\end{array}$ & $\begin{array}{c}13.3 \\
0.9732 \\
\end{array}$ & $\begin{array}{c}14.0 \\
0.9735 \\
\end{array}$ \\
\hline
\end{tabular}

The thermal degradation of the polymers was also investigated applying the Broido method in which the calculations do not depend on the reaction order. This method is performed according to $E q$. 1.6. Interestingly, the $\mathrm{E}_{\mathrm{a}}$ values from both the CR and Broido methods are in good agreement for PE-A, which supports the proposed degradation mechanism that considers the degradation of ether bonds. On the other hand, the degradation of PE-Ani-A requires higher $\mathrm{E}_{\mathrm{a}}$ to overcome the amine-linkage during the degradation process. The Broido method

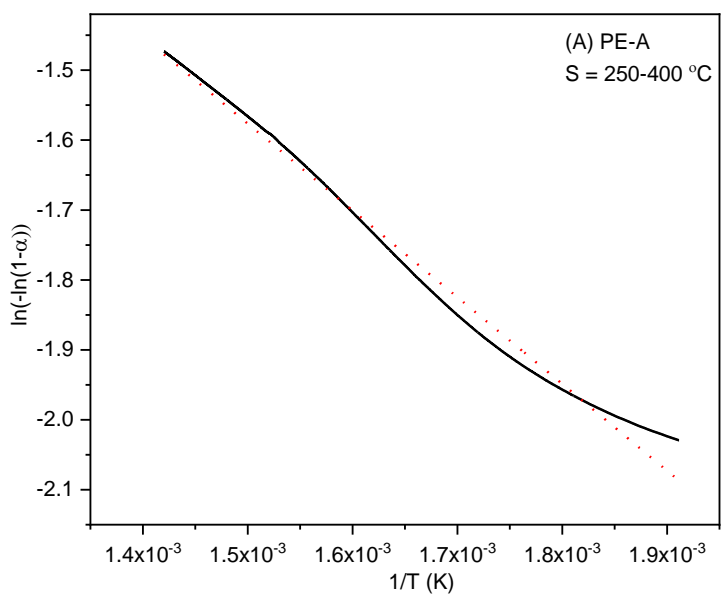

does not depend on the reaction order and assumes the reaction order $n=1$. Thus, for the reliability of the data, we can expect the reaction order to be in the range of $0.5 \leq \mathrm{n} \leq 1$ and that the thermal degradation mechanism has two steps including the dissociation of ether-bond followed by the degradation of the anilinelinkage. The fitting curves of the Broido method are shown in Figure 5. For comparison, the $E_{a}$ and $\mathrm{R}^{2}$ values from both methods are summarized in Table 3.

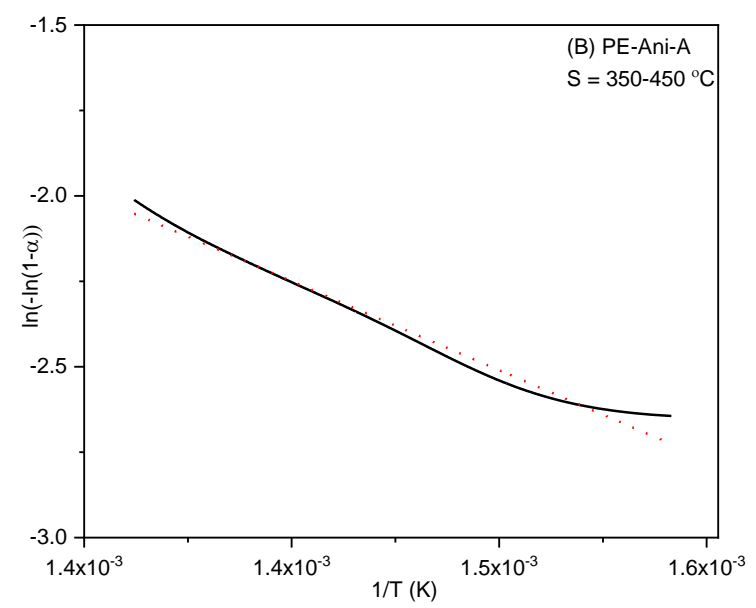

Figure 5. The Briodo model for the two polymers at the degradation stage (S); (A) PE-A and (B) PE-Ani-A. 
Table 3. The activation energy values and the $R^{2}$ values from the $C R$ and Broido models

\begin{tabular}{lccc}
\hline Polymer & $\begin{array}{c}\text { Degradation } \\
\text { Stage } \\
\left({ }^{\circ} \mathbf{C}\right)\end{array}$ & \multicolumn{2}{c}{$\begin{array}{c}\mathbf{E}_{\mathbf{a}}(\mathbf{k J} / \mathbf{m o l}) \\
\left(\mathbf{R}^{2}\right)\end{array}$} \\
\hline PE-A & S: $250-400$ & 12.0 & Broido \\
& & $(0.9842)$ & 10.3 \\
PE-Ani-A & S: $350-450$ & 24.3 & $(0.9898)$ \\
& & $(0.9908)$ & 32.7 \\
& & & $(0.9851)$ \\
\hline
\end{tabular}

\section{Conclusions}

In this study, two open-chain ether-based organic polymers were prepared and fully characterized. One of these polymers consists of bromo-substituted chains, while the other is functionalized with aniline-groups instead of the bromo-sites. Upon post-functionalization the thermal stability has been improved by $36 \%$ when compared with the parent polymer. However, this synthetic strategy has compromised the LOI value of the bromosubstituted polymer. On the other hand, the role of this modification was investigated by determining the thermal degradation kinetics parameters resulting from the $\mathrm{CR}$ and Broido models. The thermal degradation process of the two polymers follows a reaction order in the range of $0.5 \leq n \leq 1$ and the activation energy of the post-functionalized polymer is higher than that of the parent polymer. The proposed degradation mechanism suggests that, the first loss of the open-chain is related to the degradation of ether bond, while higher energy is required for the separation and degradation of the aniline group.

\section{Acknowledgment}

The authors gratefully acknowledge the Water, Environment and Arid Regions Research Center at $\mathrm{Al}$ al-Bayt University, Jordan for the polymer characterization. Many thanks are also to Tafila Technical University for supporting this project under grant number 122/2020.

\section{References}

[1] Tecklenburg, M.; Kosnak, D.; Bhatnagar, A.; Mohanty, D., J. Raman Spectrosc. 1997, 28, 755-763.

[2] Irvin, J.; Neef, C.; Kane, K.; Cassidy,P.; Tullos, G.; Clair, A., J. Polym. Sci. Part A Polym. Chem. 1992, 30, 1675-1679.

[3] Krishnan, R.; Parthiban, A., J. Polym. Res. 2013, 20, 1-30.

[4] Tkachenko I., Mol. Cryst. Liq. Cryst. 2020, 697, 85-96.

[5] Chen, S., Polymers (Basel) 2019, 11, 1-19.

[6] Qi, Y., Nat. Commun. 2019, 10, 1-9.

[7] Bourbigot, S.; Duquesne, S., J. Mater. Chem. 2007, 17, 2283-2300.

[8] Wang, C.; Liu, H.; Zhang, J.; Yang, S.; Zhang, Z.; Zhao, W., J. Anal. Appl. Pyrolysis, 2018, 134, 167-175.
[9] Osman, S.; Khattab,S.; Aly, E., J. Polym. Res. 2017, 24, 1-13.

[10] Chang, G.; Xu, Z.; Lin, R.; Luo, X., Chinese J. Chem. 2007, 25, 1207-1211.

[11] Reis, L.; Ligiéro, C.; Andrade, A.; Taylor, J.; Miranda, P., Materials (Basel), 2012, 5, 2176-2189.

[12] Hemvichian, K.; Ishida, H., Polymer (Guildf) 2002, 43, 1353-1358.

[13] Gelmont, M.; Yuzefovitch, M.; Yoffe, D.; Eden, E.; Levchik, S., Polymers (Basel), 2020, 12, 1-18.

[14] Altarawneh, S.; Max, J.; Schacher, F.; Ababneh, T., J. Polym. Res. 2020, 27, 1-19.

[15] Singh, A.; Singh, S.; Soni, P.; Mukherjee, N., J. Macromol. Sci. Part B Phys. 2020, 59, 1-24. 
[16] Alves, W.; Malmonge, J.; Mattoso, L.; De Medeiros, E., Polimeros, 2018, 28, 285292.

[17] Ma, D.; Cai, Q., Synlett. 2004, 1, 1-10.

[18] Quivelli, A.; Vitale, P.; Perna, F.; Capriati, V., Front. Chem. 2019, 7, 1-14.
[19] Altarawneh, S.; Ababneh, T.; Al-Momani, L.; Aljaafreh, I., Polym. Sci.- Ser. B, 2018, 60, 789-797.

[20] Broido, A., J. Polym. Sci. Part A-2 Polym Phys. 1969, 7, 1761-1773.

[21] Johnson, D.; Gallagher, P., J. Phys. Chem. 1972, 76, 1474-1479. 